\title{
Acute and sub-acute toxicity of Harungana madagascariensis LAM (Hypericaceae) stem bark methanol extract
}

\author{
Rebecca Madeleine Ebelle Etame ${ }^{1}$, Raymond Simplice Mouokeu ${ }^{2 *}$, Rosalie Anne Ngono Ngane ${ }^{3}$, Jean Paul Assam \\ Assam $^{1}$, Azaber Mamoune Masoohe ${ }^{1}$, Raphael Tientcheu ${ }^{1}$, Mylene Laure Hopogap ${ }^{3}$, François Xavier Etoa ${ }^{3}$ \\ ${ }^{1}$ Institute of Fisheries and Aquatic Sciences, University of Douala, Po Box 7236, Douala, Cameroon . \\ ${ }^{2}$ Laboratory of Biochemistry, Department of Biochemistry, Faculty of Science, University of Douala, Po Box 24157, Douala, Cameroon. \\ ${ }^{3}$ Department of Microbiology, University of Yaoundé I, Faculty of Sciences, Po box 812, Yaoundé, Cameroon.
}

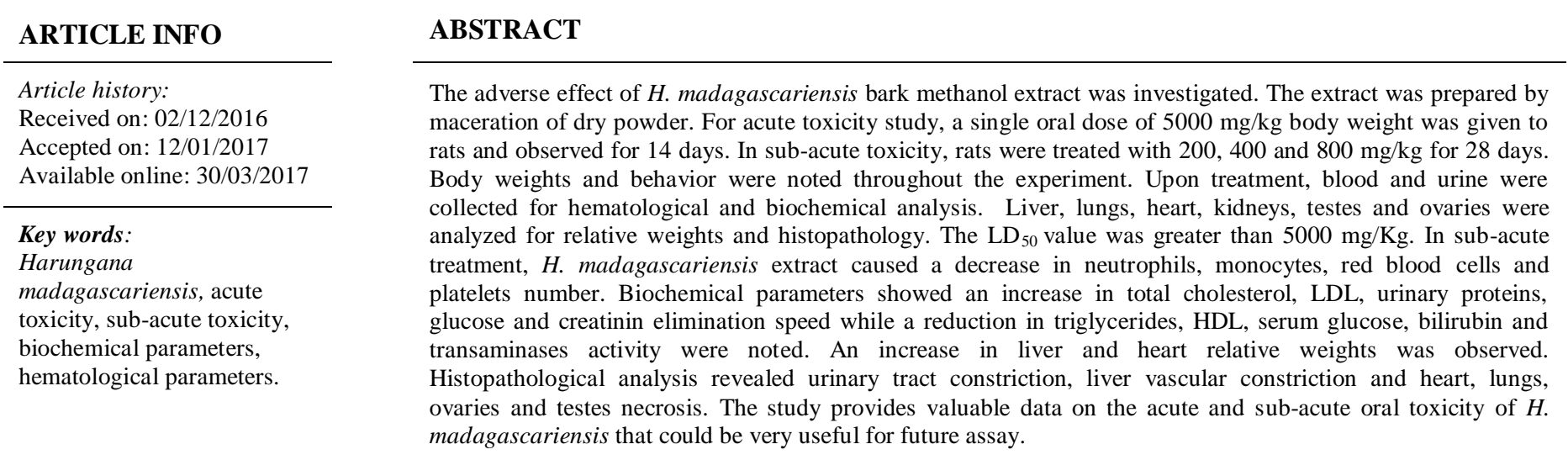

\section{INTRODUCTION}

Humans have developed knowledge and optimized the use of plants for thousands of years according to their intelligence, genius, understanding of health, diseases and relationship with the environment (Bouiddouh, 2012). These data are nowadays the basis of several scientific experiments which attest pharmacological properties of extracts derived from plants. The increase in number of users as oppose to the scarcity of scientific evidence on the safety of the medicinal plants have raised concerns regarding toxicity and detrimental effects of

\footnotetext{
* Corresponding Author

Raymond Simplice MOUOKEU, Institute of Fisheries and Aquatic Sciences, University of Douala, Po Box 7236, Douala, Cameroon. Email: moraysi@yahoo.fr
}

these remedies. There is now growing evidence that many herbal medicines do cause serious toxicity to their users (Aguianaga et al., 2014; Naidu et al., 2014). Although the pharmacological properties of plant extracts are continuously been established, their safety must be studied thoroughly to maximize their benefits to mankind. H. madagascariensis is a well-known plant in Cameroon where different parts are highly valued for the treatment of diverse human diseases including anemia, jaundice, bleeding, gonorrhea, malaria, asthma, liver diseases, diabetes, pancreatic and biliary problems (Nicolas, 2012; Adeneye et al., 2008). Previous studies on this plant involving bark or leaves revealed antihelminthiase properties (Koné and Kamanzi, 2006), antimalaria (Manzo, 2012), antidiabetic (Mangambu, 2014) and antimicrobial activities (Etchike et al., 2011). The antimicrobial properties of $H$. madagascariensis stem bark of methanol extract are well known. 
However, the extract has not yet been subject to toxicological studies. Recent studies revealed side effects of the leaves extract of this plant on liver cells and lipid metabolism. The present study was therefore designed to investigate the safety of methanol extract from stem bark of $H$. madagascariensis.

\section{MATERIALS AND METHODS}

\section{Plant material}

H. madagascariensis bark used in the experiment was collected at Kalla's mountain in June 2014, in the Centre Region of Cameroon. The identification of this plant was done at the National Herbarium of Cameroon, compared to the reference sample number $4224 \mathrm{HNC}$.

\section{Experimental animals}

Twenty female Wistar rats aged between 8 and 12 weeks, weighing 100-150 g were used for acute toxicity study. For subacute toxicity study, fifty Wistar rats (25 males and 25 females) aged between 8 and 12 weeks, weighing 150-200 g were used. All these animals were purchased at the Laboratory of Animal Biology, University of Yaoundé I and acclimatized for two weeks prior to experiments. These animals were fed daily with a standard rat diet. They had free access to drinking water and were maintained at 12 hours cycle day and night conditions. The animals were kept in a room with temperature maintained at $22 \pm 2{ }^{\circ} \mathrm{C}$. The studies were conducted according to the ethical guidelines of Committee for Control and Supervision of Experiments on Animals (Registration no. 173/CPCSEA, dated 28 January, 2000), Government of India, on the use of animals for scientific research.

\section{Plant extract preparation}

H. madagascariensis barks once collected was cut into small pieces, dried 30 days at room temperature and then ground to yield a powder. $250 \mathrm{~g}$ of powder was macerated in $250 \mathrm{ml}$ methanol over 48 hours, filtered through Whatman paper $\mathrm{N}^{\circ} 1$ and further concentrated using rotary evaporator at $65^{\circ} \mathrm{C}$. The extract obtained was placed in an oven 48 hours at $40^{\circ} \mathrm{C}$ to remove residual solvent, then weighed and stored at $4^{\circ} \mathrm{C}$.

\section{Acute toxicity study}

The oral acute toxicity of $H$. madagascariensis bark extract was performed according to OCDE protocols $\left(\mathrm{OCDE}_{\mathrm{a}}\right.$, 2008). The test was performed on 10 rats divided into two groups of 5 animals, comprising one control and one test group. These animals were fasted 15 hours then weighed and treated by esophageal gavage. Animals in the control group received 5\% dimethylsulfoxide (DMSO) and those of the test group were given a unique dose of $H$. madagascariensis extract; $5000 \mathrm{mg} / \mathrm{kg}$ b.w. Upon treatment, these animals were fasted for additional 3 hours. During this period, signs of toxicity including sensitivity to pain, noise, tail state, stool appearance and mobility were noted. Furthermore, death was monitored 14 days following treatment.

\section{Sub-acute toxicity study Experimental design}

The sub-acute toxicity study was conducted according to the OCDE guidelines $\left(\mathrm{OCDE}_{\mathrm{b}}, 2008\right)$. Briefly, 50 rats, including 25 males and 25 females were divided into 5 groups of 10 animals. Group 1; control group received 5\% DMSO. Group II and III received 200 and $400 \mathrm{mg} / \mathrm{Kg}$ b.w plant extract respectively. Group IV and $\mathrm{V}$ received $800 \mathrm{mg} / \mathrm{Kg}$ b.w extract. The administration was done daily over a period of 28 days by esophageal gavage for Group I to Group IV. Following 28 days plant extract administration, Group V (satellite) was fed under the same conditions for additional 15 days. During the experiment, animals were weighted every two days and signs of toxicity were observed weekly.

\section{Sample preparation}

Animals were subjected to 24 hours food and water fasting and urines were collected, centrifuged at $3000 \mathrm{rpm}$ for 10 minutes and stored at $-80^{\circ} \mathrm{C}$. They were further anesthetized intraperitoneally with ketamine $(50 \mathrm{mg} / \mathrm{kg}$ ) and blood was collected into EDTA and dry tubes by cardiac puncture. Blood in dry tubes was allowed to clot for 6 hours then centrifuged at 3000 rpm for 15 minutes. The serum was removed and stored at $-80^{\circ} \mathrm{C}$ for biochemical assays.

Animals were further dissected and organs such as liver, heart, kidney, lung, testes ovaries collected, weighed and relative organ weights were evaluated. Part of each organ was used for protein analysis and maintained at ambient conditions in 9\% formalin- $\mathrm{NaCl}$ solution for histological analysis.

\section{Hematological analysis}

Hematological parameters; white blood cells, red blood cells, hematocrit, platelets, hemoglobin, neutrophils, basophils, eosinophils, lymphocytes, monocytes, mean corpuscular hemoglobin $(\mathrm{MCH})$, Mean Corpuscular Hemoglobin Concentration (MCHC) and Mean Corpuscular Volume (MCV) were analyzed using automaton hematology (PENTRA X 80).

\section{Biochemical parameter analysis}

Biochemical parameters including creatinin clearance, glucose, proteins, ALT, AST, total cholesterol, triglycerides, HDL cholesterol, LDL cholesterol, total bilirubin and urea were analyzed in serum while urea, creatinin, glucose and protein concentration were evaluated in urine. These analyses were performed using commercial kits (Hospitex Diagnostics std, Roma, Italia).

\section{Histological analysis}

A piece of each organ was fixed in $10 \%$ formalin and embedded in paraffin. 5 to $6 \mathrm{~mm}$ portion of each piece was routinely stained with haematoxylin and eosin, and examined under a light microscope (Olympus CH02) (Mouokeu et al., 2011). Any alteration relative to control was noted. 


\section{Statistical analysis}

Results were presented as mean \pm standard deviation. For each parameter, the one way ANOVA was used to detect significant differences between groups, followed by Waller Duncan test $(\mathrm{p}<0.05)$.

\section{RESULTS}

\section{Acute toxicity}

Rats treated with the methanol stem bark extract of $H$. madagascariensis showed behavior change including decreased in sensitivity to pain, noise and locomotion. No death was noticed within the treated animals. The oral $\mathrm{LD}_{50}$ of $H$. madagascariensis methanol stem bark extract is therefore greater than $5000 \mathrm{mg} / \mathrm{Kg}$ b.w.

\section{Sub-acute toxicity \\ Body weights}

The $H$. madagascariensis extract in sub-acute administration caused weight loss in animals of both sexes at 800 $\mathrm{mg} / \mathrm{kg}$ (data not shown).

\section{Relative organ weight}

H. madagascariensis methanol extract in sub-acute treatment over 28 days revealed no change in the relative weight of kidney, lung and ovaries (Table 1). However, there was an increase in relative liver $(200 \mathrm{mg} / \mathrm{Kg})$, heart $(800 \mathrm{mg} / \mathrm{kg})$ and testes $(400 \mathrm{mg} / \mathrm{kg})$ weight.

\section{Hematological parameters}

H. madagascariensis methanol extract in sub-acute treatment induced an increase in white blood cells in males and females (200 mg/kg), Mean Corpuscular Volume (MCV), eosinophils and a decreased in red blood cell, platelet content, neutrophils and monocytes from $200 \mathrm{mg} / \mathrm{kg}$ in both sexes. However, no effect on hematocrit, hemoglobin, Mean Corpuscular Hemoglobin $(\mathrm{MCH})$ and Mean Corpuscular Hemoglobin Concentration (MCHC) regardless of dose and sex was observed. Decrease in neutrophils tends to standardize 15 days after treatment.

\section{Biochemical parameters}

Daily oral sub-acute treatment with $H$. madagascariensis extract induced an increase in total cholesterol, LDL cholesterol (in both males and females from $400 \mathrm{mg} / \mathrm{kg}$ ) and a decrease in HDL cholesterol and triglycerides concentrations from $400 \mathrm{mg} / \mathrm{kg}$ (Table 3). In addition, the extract caused a decrease in ALT and AST activity, total bilirubin concentration and an increase in serum protein levels in animals of both sexes at 200 and 400 $\mathrm{mg} / \mathrm{kg}$.

In addition, there was an increase in creatinin clearance, serum glucose, urinary protein concentration and a decrease in urea serum level in animals of both sexes. This increase was associated with increased urinary excretion of glucose and protein. In addition, the extract led to decrease kidney, heart, lungs, testes and ovaries protein levels in male and female. However, total cholesterol, HDL cholesterol, urine and serum glucose concentrations tend to become normal 15 days following treatment.

\section{Histological studies}

Histological analysis of rat organs treated with $H$. madagascariensis extract revealed early vascular congestions of the liver, more developed in females than in males. At the level of the kidney, a narrowing of the urinary tract and expansion of mesangium was observed. In addition, necrosis of the cardiac muscle fibers, lungs, ovaries and testes were also noted (Figure1).

Table 1: relative weights (mg/g b.w.) of rat's organs in sub-chronic toxicity of methanol extract of bark of H. madagascariensis as a function of doses and sexes.

\begin{tabular}{|c|c|c|c|c|c|c|}
\hline Sex & Doses (mg/kg) & Liver & kidney & Heart & Lung & Testicules/ovaries \\
\hline \multirow{5}{*}{$\stackrel{\frac{0}{\pi}}{\sum}$} & Control & $2.42 \pm 0.17^{\mathrm{a}}$ & $0.30 \pm 0.04^{\mathrm{a}}$ & $0.35 \pm 0.03^{b}$ & $1.02 \pm 0.25^{\mathrm{a}}$ & $0.44 \pm 0.10^{\mathrm{b}}$ \\
\hline & 200 & $2.53 \pm 0.21^{\mathrm{a}}$ & $0.28 \pm 0.17^{\mathrm{a}}$ & $0.34 \pm 0.04^{\mathrm{b}}$ & $0.86 \pm 0.09^{\mathrm{a}}$ & $0.39 \pm 0.17^{\mathrm{b}}$ \\
\hline & 400 & $2.48 \pm 0.28^{\mathrm{a}}$ & $0.27 \pm 0.01^{\mathrm{a}}$ & $0.36 \pm 0.02^{b}$ & $1.04 \pm 0.23^{\mathrm{a}}$ & $0.72 \pm 0.03^{\mathrm{a}}$ \\
\hline & 800 & $2.23 \pm 0.81^{\mathrm{a}}$ & $0.28 \pm 0.01^{\mathrm{a}}$ & $0.38 \pm 0.1^{\mathrm{ab}}$ & $0.87 \pm 0.14^{\mathrm{a}}$ & $0.40 \pm 0.12^{\mathrm{b}}$ \\
\hline & Satellite & $2.85 \pm 0.72^{\mathrm{a}}$ & $0.34 \pm 0.17^{\mathrm{a}}$ & $0.40 \pm 0.09^{\mathrm{a}}$ & $0.94 \pm 0.14^{\mathrm{a}}$ & $0.43 \pm 0.16^{\mathrm{b}}$ \\
\hline \multirow{5}{*}{ 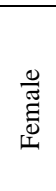 } & Control & $2.99 \pm 0.66^{\mathrm{b}}$ & $0.31 \pm 0.02^{\mathrm{a}}$ & $0.39 \pm 0.03^{\mathrm{a}}$ & $0.90 \pm 0.24^{\mathrm{a}}$ & $0.03 \pm 0.00^{\mathrm{a}}$ \\
\hline & 200 & $4.01 \pm 0.34^{\mathrm{a}}$ & $0.31 \pm 0.03^{\mathrm{a}}$ & $0.40 \pm 0.00^{\mathrm{a}}$ & $0.79 \pm 0.08^{\mathrm{a}}$ & $0.04 \pm 0.01^{\mathrm{a}}$ \\
\hline & 400 & $2.84 \pm 0.11^{b}$ & $0.31 \pm 0.00^{\mathrm{a}}$ & $0.38 \pm 0.04^{\mathrm{a}}$ & $1.04 \pm 0.27^{\mathrm{a}}$ & $0.03 \pm 0.00^{\mathrm{a}}$ \\
\hline & 800 & $3.04 \pm 0.04^{\mathrm{ab}}$ & $0.35 \pm 0.00^{\mathrm{a}}$ & $0.42 \pm 0.01^{\mathrm{a}}$ & $0.89 \pm 0.13^{\mathrm{a}}$ & $0.03 \pm 0.00^{\mathrm{a}}$ \\
\hline & Satellite & $2.49 \pm 0.06^{\mathrm{b}}$ & $0.32 \pm 0.04^{\mathrm{a}}$ & $0.39 \pm 0.03^{\mathrm{a}}$ & $0.95 \pm 0.05^{\mathrm{a}}$ & $0.03 \pm 0.00^{\mathrm{a}}$ \\
\hline
\end{tabular}

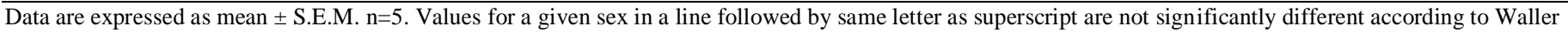
Duncan's multiple comparison test $(\mathrm{P}<0.05)$. 
Table 2: Hematological parameters of rats in sub-chronic toxicity of methanol extract of $H$. madagascariensis as a function of doses and sexes.

\begin{tabular}{|c|c|c|c|c|c|c|}
\hline \multirow{14}{*}{$\frac{0}{\Sigma}$} & & DMSO & 200 & 400 & 800 & Satellite \\
\hline & $\mathrm{WBC}\left(\right.$ cells $\left./ \mathrm{mm}^{3}\right)$ & $6560.00 \pm 1971.80^{\mathrm{c}}$ & $18320.00 \pm 2629.06^{\mathrm{a}}$ & $15960.00 \pm 1485.93^{\mathrm{ab}}$ & $13760.00 \pm 328.63^{b}$ & $9000.00 \pm 2738.61^{c}$ \\
\hline & $\mathrm{RBC}\left(\right.$ cells $\left./ \mathrm{mm}^{3}\right) \times 10^{6}$ & $5.33 \pm 65612.49^{\mathrm{a}}$ & $4.89 \pm 547.72^{\mathrm{b}}$ & $4.87 \pm 1.02^{\mathrm{b}}$ & $4.96 \pm 43280.48^{\mathrm{b}}$ & $4.72 \pm 4.79^{\mathrm{b}}$ \\
\hline & Hematocrit (\%) & $47.60 \pm 5.68^{\mathrm{a}}$ & $45.60 \pm 3.28^{\mathrm{a}}$ & $48.8 \pm 4.816^{\mathrm{a}}$ & $47.00 \pm 3.46^{\mathrm{a}}$ & $45.6 \pm 2.88^{\mathrm{a}}$ \\
\hline & Plaquette (cells $/ \mathrm{mm}^{3}$ ) & $651800.00 \pm 53129.08^{\mathrm{a}}$ & $153200.00 \pm 836.66^{\mathrm{b}}$ & $156400.00 \pm 4929.50^{\mathrm{b}}$ & $160000.00 \pm 0.00^{\mathrm{b}}$ & $152000.00 \pm 1870.82^{\mathrm{b}}$ \\
\hline & Hemoglobin $(\mathrm{g} / \mathrm{dl})$ & $16.42 \pm 1.28^{\mathrm{a}}$ & $15.46 \pm 0.50^{\mathrm{a}}$ & $14.94 \pm 0.3286^{\mathrm{a}}$ & $15.66 \pm 1.16^{\mathrm{a}}$ & $15.18 \pm 0.98^{\mathrm{a}}$ \\
\hline & Neutrophils (cells $/ \mathrm{mm}^{3}$ ) & $18132.00 \pm 1522.66^{\mathrm{a}}$ & $5545.60 \pm 534.57^{\mathrm{c}}$ & $7600.00 \pm 646.31^{\mathrm{b}}$ & $6600.00 \pm 919.02^{\mathrm{bc}}$ & $16579.20 \pm 1397.78^{\mathrm{a}}$ \\
\hline & Basophils (cells/mm³) & $0.00 \pm 0.00^{\mathrm{a}}$ & $0.00 \pm 0.00^{\mathrm{a}}$ & $0.00 \pm 0.00^{\mathrm{a}}$ & $0.00 \pm 0.00^{\mathrm{a}}$ & $0.00 \pm 0.00^{\mathrm{a}}$ \\
\hline & Eosinophils $\left(\right.$ cells $\left./ \mathrm{mm}^{3}\right)$ & $65.60 \pm 19.71^{\mathrm{d}}$ & $276.80 \pm 70.10^{\mathrm{a}}$ & $148.40 \pm 19.71^{\mathrm{b}}$ & $137.60 \pm 3.28^{\mathrm{bc}}$ & $90.00 \pm 27.38^{\text {cd }}$ \\
\hline & Lymphocytes $\left(\right.$ cells $\left./ \mathrm{mm}^{3}\right)$ & $5424.00 \pm 131.45^{\mathrm{c}}$ & $6456.00 \pm 142.40^{\mathrm{bc}}$ & $6984.00 \pm 1971.80^{\mathrm{b}}$ & $10608.00 \pm 43.81^{\mathrm{a}}$ & $6960.00 \pm 0.00^{\mathrm{b}}$ \\
\hline & Monocytes (cells/mm³) & $244.00 \pm 58.56^{\mathrm{a}}$ & $121.60 \pm 39.55^{\mathrm{bc}}$ & $155.60 \pm 19.71^{\mathrm{b}}$ & $137.60 \pm 3.28^{\mathrm{bc}}$ & $90.00 \pm 27.38^{\mathrm{c}}$ \\
\hline & $\mathrm{MCH}(\mathrm{pg})$ & $29.60 \pm 0.54^{\mathrm{a}}$ & $30.40 \pm 0.54^{\mathrm{a}}$ & $29.40 \pm 2.19^{\mathrm{a}}$ & $30.40 \pm 0.54^{\mathrm{a}}$ & $30.20 \pm 0.83^{\mathrm{a}}$ \\
\hline & $\operatorname{MCHC}(\mathrm{g} / \mathrm{dl})$ & $33.32 \pm 0.10^{\mathrm{a}}$ & $33.18 \pm 0.16^{\mathrm{a}}$ & $33.24 \pm 0.05^{\mathrm{a}}$ & $33.28 \pm 0.10^{\mathrm{a}}$ & $33.25 \pm 0.21^{\mathrm{a}}$ \\
\hline & $\mathrm{MCV}(\mathrm{fl})$ & $87.60 \pm 0.54^{\mathrm{b}}$ & $92.80 \pm 1.09^{\mathrm{a}}$ & $92.00 \pm 0.00^{\mathrm{a}}$ & $91.40 \pm 1.34^{\mathrm{a}}$ & $91.20 \pm 1.09^{\mathrm{a}}$ \\
\hline \multirow{13}{*}{ 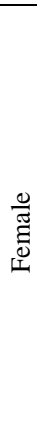 } & WBC $\left(\right.$ cells $\left./ \mathrm{mm}^{3}\right)$ & $11400.00 \pm 979.79^{\mathrm{c}}$ & $19280.00 \pm 657.26^{\mathrm{a}}$ & $11200.00 \pm 1095.44^{\mathrm{c}}$ & $15200.00 \pm 1643.16^{b}$ & $16000.00 \pm 0.00^{b}$ \\
\hline & $\mathrm{RBC}\left(\right.$ cells $\left./ \mathrm{mm}^{3}\right) \times 10^{6}$ & $6.02 \pm 2.93^{\mathrm{a}}$ & $6.17 \pm 3.85^{\mathrm{a}}$ & $5.76 \pm 1.07^{\mathrm{ab}}$ & $5.33 \pm 3.01^{\mathrm{ab}}$ & $4.94 \pm 3.80^{\mathrm{b}}$ \\
\hline & Hematocrit (\%) & $47.00 \pm 5.61^{\mathrm{ab}}$ & $53.2 \pm 1.64^{\mathrm{a}}$ & $51.2 \pm 6.57^{\mathrm{a}}$ & $47.8 \pm 1.64^{\mathrm{ab}}$ & $44.6 \pm 2.19^{\mathrm{ab}}$ \\
\hline & Plaquette (cells $/ \mathrm{mm}^{3}$ ) & $282000.00 \pm 10954.45^{\mathrm{a}}$ & $154000.00 \pm 447.21^{\mathrm{b}}$ & $155000.00 \pm 1095.44^{\mathrm{b}}$ & $161000.00 \pm 8763.56^{\mathrm{b}}$ & $153000.00 \pm 2738.61^{\mathrm{b}}$ \\
\hline & Hemoglobin $(\mathrm{g} / \mathrm{dl})$ & $16.54 \pm 0.21^{\mathrm{ab}}$ & $17.46 \pm 0.55^{\mathrm{a}}$ & $16.24 \pm 2.24^{\mathrm{ab}}$ & $15.90 \pm 0.54^{\mathrm{ab}}$ & $14.86 \pm 0.76^{\mathrm{b}}$ \\
\hline & Neutrophils (cells $/ \mathrm{mm}^{3}$ ) & $5240.00 \pm 54.77^{\mathrm{a}}$ & $4204.80 \pm 280.43^{\mathrm{b}}$ & $1843.20 \pm 324.25^{\mathrm{d}}$ & $3140.00 \pm 350.54^{\mathrm{c}}$ & $5462.40 \pm 468.85^{\mathrm{a}}$ \\
\hline & Basophils (cells/mm³) & $0.00 \pm 0.00^{\mathrm{a}}$ & $0.00 \pm 0.00^{\mathrm{a}}$ & $0.00 \pm 0.00^{\mathrm{a}}$ & $0.00 \pm 0.00^{\mathrm{a}}$ & $0.00 \pm 0.00^{\mathrm{a}}$ \\
\hline & Eosinophils $\left(\right.$ cells $/ \mathrm{mm}^{3}$ ) & $221.60 \pm 8.76^{\mathrm{a}}$ & $82.00 \pm 16.43^{\mathrm{d}}$ & $116.00 \pm 10.95^{\mathrm{c}}$ & $152.00 \pm 16.43^{\mathrm{b}}$ & $163.60 \pm 3.28^{\mathrm{b}}$ \\
\hline & Lymphocytes $\left(\right.$ cells $\left./ \mathrm{mm}^{3}\right)$ & $8162.40 \pm 686.84^{\mathrm{bc}}$ & $5260.00 \pm 492.95^{\mathrm{d}}$ & $8984.00 \pm 1194.03^{\mathrm{bc}}$ & $11792.00 \pm 1960.84^{\mathrm{a}}$ & $10452.80 \pm 146.78^{\mathrm{ab}}$ \\
\hline & Monocytes (cells/mm³) & $240.80 \pm 17.52^{\mathrm{a}}$ & $82.00 \pm 16.43^{\mathrm{d}}$ & $105.20 \pm 1.30^{\mathrm{c}}$ & $152.00 \pm 16.43^{\mathrm{b}}$ & $162.48 \pm 3.28^{\mathrm{b}}$ \\
\hline & $\mathrm{MCH}(\mathrm{pg})$ & $28.20 \pm 0.83^{\mathrm{a}}$ & $27.40 \pm 0.54^{\mathrm{a}}$ & $28.20 \pm 1.09^{\mathrm{a}}$ & $29.00 \pm 1.00^{\mathrm{a}}$ & $29.00 \pm 1.00^{\mathrm{a}}$ \\
\hline & $\operatorname{MCHC}(\mathrm{g} / \mathrm{dl})$ & $33.22 \pm 0.14^{\mathrm{a}}$ & $33.20 \pm 0.08^{\mathrm{a}}$ & $33.22 \pm 0.00^{\mathrm{a}}$ & $33.15 \pm 0.00^{\mathrm{a}}$ & $33.23 \pm 0.00^{\mathrm{a}}$ \\
\hline & MCV (fl) & $86.60 \pm 1.34^{\mathrm{a}}$ & $85.80 \pm 1.64^{\mathrm{a}}$ & $85.80 \pm 3.83^{\mathrm{a}}$ & $89.60 \pm 2.19^{\mathrm{a}}$ & $89.20 \pm 4.38^{\mathrm{a}}$ \\
\hline
\end{tabular}

Data are expressed as mean \pm S.E.M. $n=5$. Values for a given sex in a line followed by same letter as superscript are not sign ificantly different according to Waller Duncan's multiple comparison test $(P<0.05)$. MCH= mean corpuscular hemoglobin, MCHC $=$ Mean Corpuscular Hemoglobin Concentration MCV =Mean Corpuscular Volume
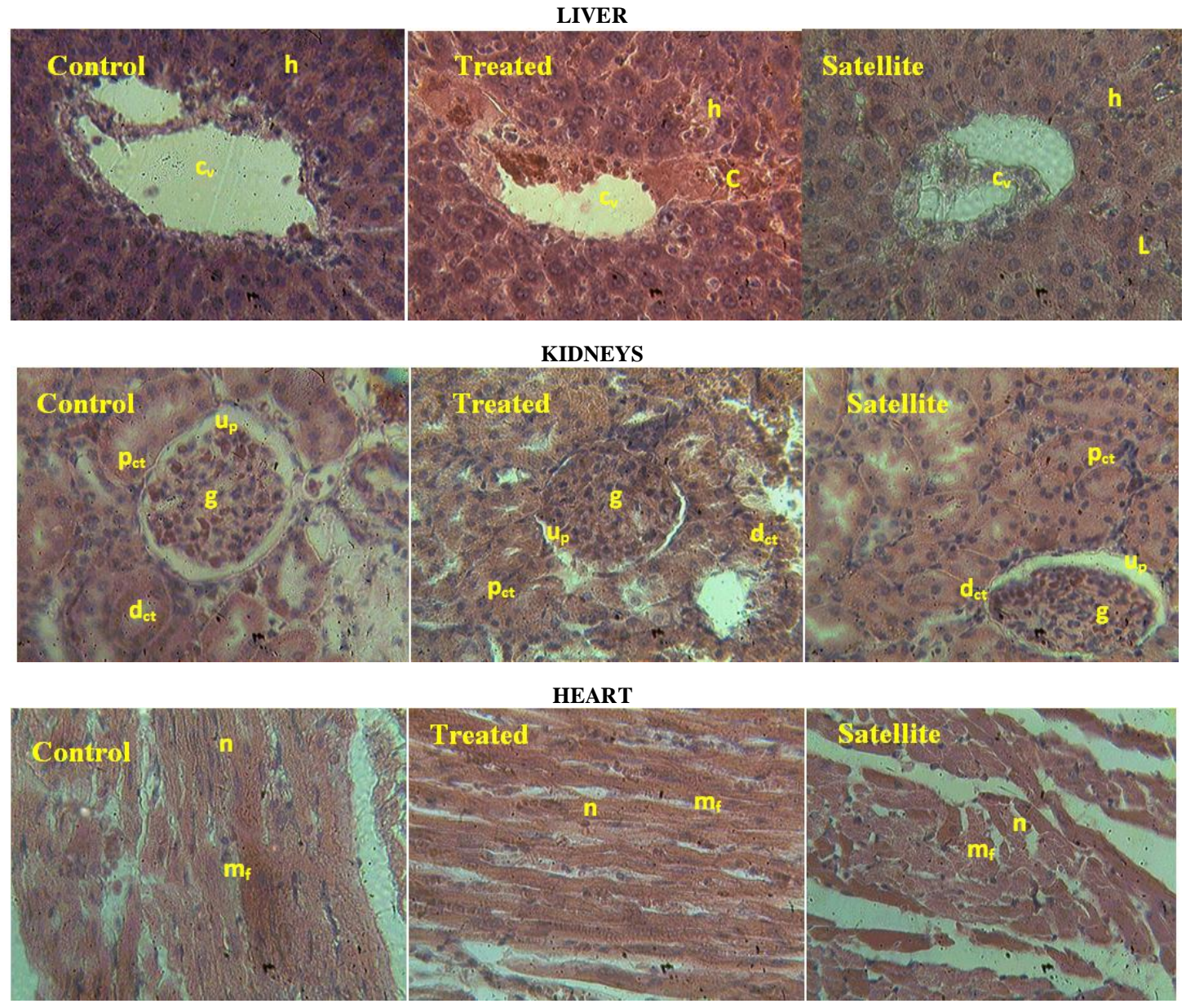


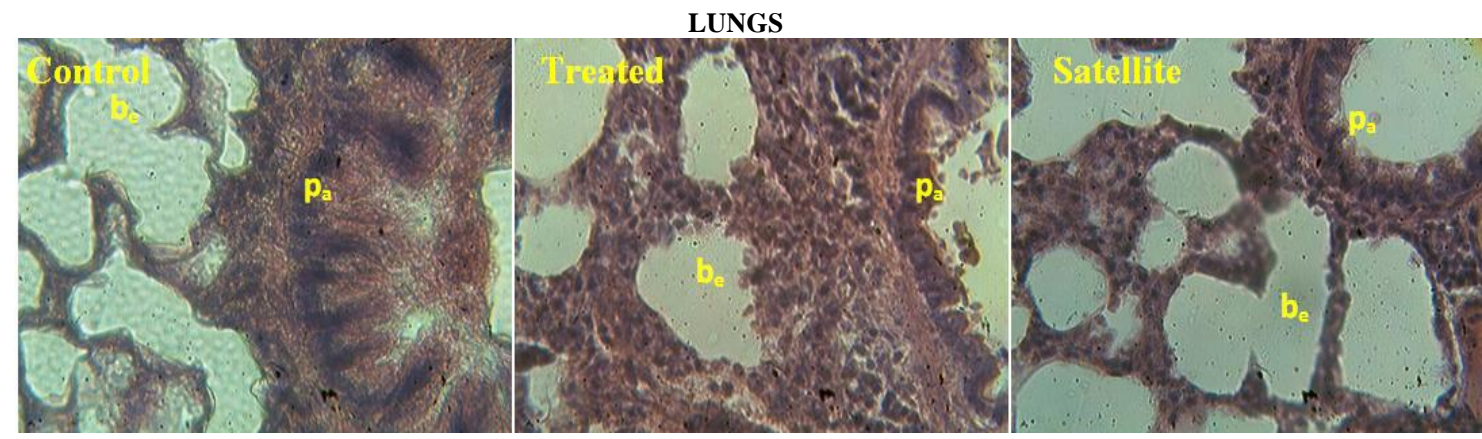

TESTES

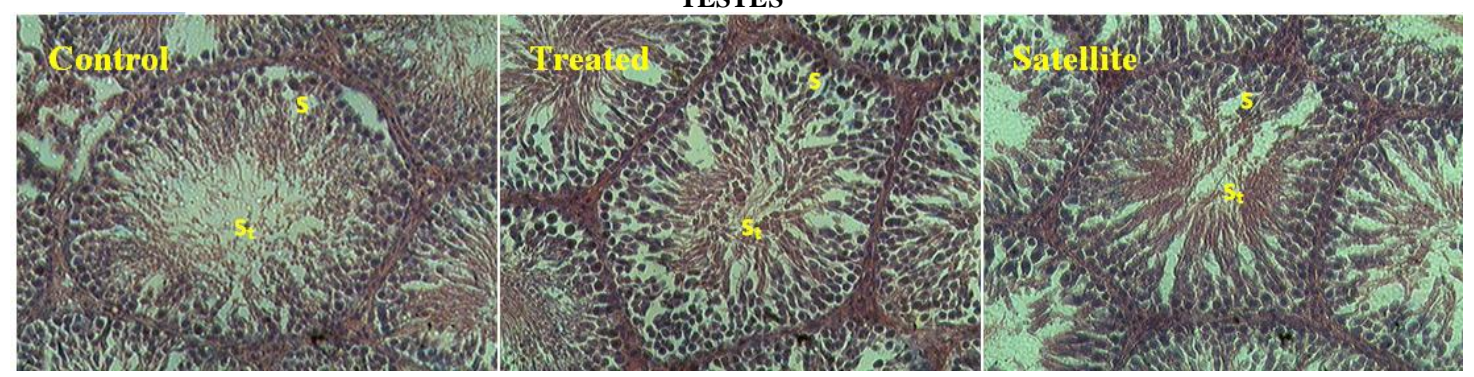

OVARIES

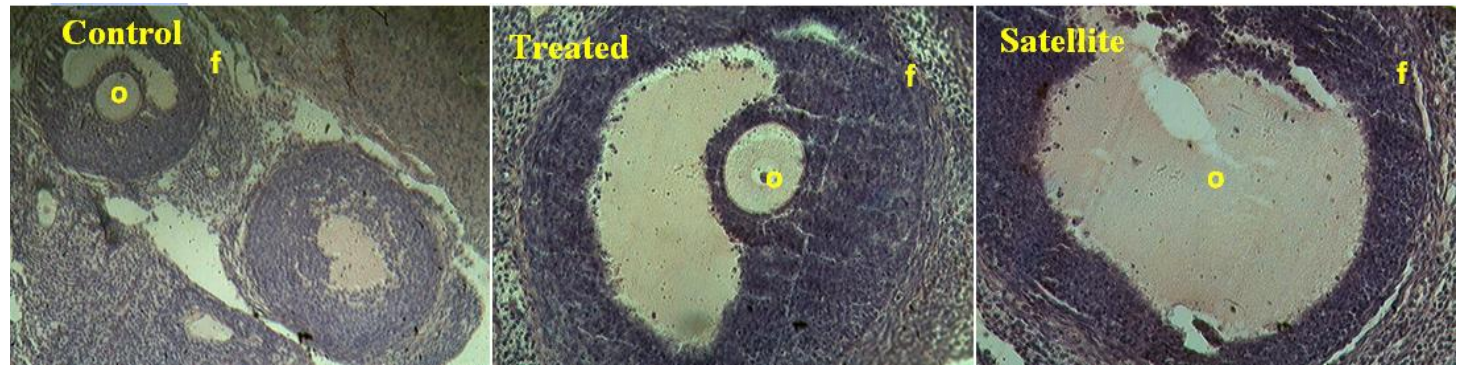

The organs of the rats exposed to H. madagascariensis extract showed: Light vacuole-like space (L) and congestions in liver(C); congestion of glomeruli and widening of the urinary space, normal heart, lungs, testes and ovary. Histological analysis of the organs of the control rats showed normal structure: hepatocytes $(h)$, central veine $\left(\mathrm{c}_{\mathrm{v}}\right)$, bronchial epithelium $\left(\mathrm{b}_{\mathrm{e}}\right)$, glomerular $(\mathrm{g})$, distal convoluted tubules $\left(\mathrm{d}_{\mathrm{ct}}\right)$, proximal convoluted tubuled $\left(\mathrm{p}_{\mathrm{ct}}\right)$, urinary space $\left(\mathrm{u}_{\mathrm{p}}\right)$ membrane, muscle fiber $\left(\mathrm{m}_{\mathrm{f}}\right)$, nuclei $(\mathrm{n})$, spermatozoa $(\mathrm{s})$, seminiferous tubules $\left(\mathrm{s}_{\mathrm{t}}\right)$, follicle $(\mathrm{f})$, oocyte $(\mathrm{o})$

Fig. 1: Histology of rats organs exposed to sub-acute oral treatment of $\mathrm{H}$. madagascariensis methanol stem bark extract

Table 3: Biochemical parameters of rats in sub-acute toxicity of methanol stem bark extract of $H$. madagascariensis.

\begin{tabular}{|c|c|c|c|c|c|c|}
\hline & Dose (mg/Kg) & Control & 200 & 400 & 800 & Satellite \\
\hline \multirow{20}{*}{$\frac{\frac{0}{\sigma \sigma}}{\Sigma}$} & Serum creatinine $(\mathrm{mg} / \mathrm{dl})$ & $2168.29 \pm 430.65^{\mathrm{a}}$ & $1490.00 \pm 138.88^{\mathrm{b}}$ & $2112.70 \pm 77.04^{\mathrm{a}}$ & $1968.14 \pm 141.52^{\mathrm{ab}}$ & $2001.50 \pm 0.00^{\mathrm{a}}$ \\
\hline & Creatinine clearance $(\mathrm{mg} / \mathrm{dl})$ & $0.0021 \pm 0.00022^{\mathrm{ab}}$ & $0.0033 \pm 0.00014^{\mathrm{ab}}$ & $0.0045 \pm 0.00018^{\mathrm{a}}$ & $0.0057 \pm 0.00433^{\mathrm{a}}$ & $0.0017 \pm 0.00002^{\mathrm{b}}$ \\
\hline & Total cholesterol $(\mathrm{mg} / \mathrm{dl})$ & $60.81 \pm 5.46^{\mathrm{b}}$ & $57.37 \pm 9.11^{\mathrm{b}}$ & $77.97 \pm 4.70^{\mathrm{a}}$ & $73.07 \pm 6.43^{\mathrm{ab}}$ & $79.59 \pm 11.47^{\mathrm{a}}$ \\
\hline & HDL cholesterol $(\mathrm{mg} / \mathrm{dl})$ & $31.35 \pm 3.58^{\mathrm{a}}$ & $24.01 \pm 5.92^{\mathrm{ab}}$ & $20.62 \pm 2.55^{\mathrm{b}}$ & $22.57 \pm 1.52^{\mathrm{ab}}$ & $28.96 \pm 0.91^{\text {ab }}$ \\
\hline & LDL cholesterol (mg/dl) & $16.47 \pm 0.19^{\mathrm{c}}$ & $18.60 \pm 1.29^{\mathrm{c}}$ & $47.74 \pm 2.72^{\mathrm{a}}$ & $30.76 \pm 1.08^{\mathrm{b}}$ & $28.41 \pm 0.89^{\mathrm{b}}$ \\
\hline & Triglycerides (mg/dl) & $68.25 \pm 3.38^{\mathrm{a}}$ & $69.90 \pm 5.65^{\mathrm{a}}$ & $61.25 \pm 2.85^{\mathrm{a}}$ & $41.93 \pm 3.28^{\mathrm{b}}$ & $28.76 \pm 10.50^{c}$ \\
\hline & ALT (U/L) & $44.81 \pm 2.67^{\mathrm{a}}$ & $32.80 \pm 4.06^{\mathrm{b}}$ & $18.62 \pm 1.11^{\mathrm{c}}$ & $34.92 \pm 2.46^{\mathrm{b}}$ & $45.98 \pm 3.63^{\mathrm{a}}$ \\
\hline & AST (U/L) & $58.49 \pm 3.70^{\mathrm{b}}$ & $23.57 \pm 6.17^{\mathrm{c}}$ & $48.01 \pm 8.64^{\mathrm{b}}$ & $80.12 \pm 0.63^{\mathrm{a}}$ & $76.47 \pm 1.95^{\mathrm{a}}$ \\
\hline & Serum protein $(\mathrm{mg} / \mathrm{dl})$ & $5.01 \pm 0.58^{\mathrm{c}}$ & $6.58 \pm 0.97^{\mathrm{b}}$ & $6.64 \pm 0.12^{\mathrm{b}}$ & $8.28 \pm 0.38^{\mathrm{a}}$ & $6.82 \pm 0.13^{\mathrm{b}}$ \\
\hline & Urinary protein $(\mathrm{mg} / \mathrm{dl})$ & $0.08 \pm 0.00^{\mathrm{b}}$ & $0.08 \pm 0.001^{\mathrm{b}}$ & $0.10 \pm 0.00^{\mathrm{a}}$ & $0.06 \pm 0.00^{\mathrm{c}}$ & $0.07 \pm 0.00^{\mathrm{b}}$ \\
\hline & Serum glucose $(\mathrm{mg} / \mathrm{dl})$ & $29.16 \pm 7.01^{\mathrm{b}}$ & $29.36 \pm 2.24^{\mathrm{b}}$ & $32.53 \pm 5.05^{\mathrm{ab}}$ & $42.85 \pm 5.76^{\mathrm{a}}$ & $41.04 \pm 1.59^{\mathrm{a}}$ \\
\hline & Urinary glucose $(\mathrm{mg} / \mathrm{dl})$ & $40.79 \pm 5.67^{\mathrm{c}}$ & $51.66 \pm 1.29^{\mathrm{b}}$ & $70.23 \pm 7.60^{\mathrm{a}}$ & $68.05 \pm 4.81^{\mathrm{a}}$ & $65.49 \pm 11.51^{\mathrm{a}}$ \\
\hline & Serum urea (mg/dl) & $55.51 \pm 22.95^{\mathrm{bc}}$ & $72.06 \pm 11.33^{\mathrm{c}}$ & $59.31 \pm 13.22^{\mathrm{ab}}$ & $40.00 \pm 12.27^{\mathrm{c}}$ & $18.96 \pm 4.72^{\mathrm{d}}$ \\
\hline & Urinary urea $(\mathrm{mg} / \mathrm{dl})$ & $596.20 \pm 35.84^{\mathrm{c}}$ & $863.79 \pm 9.44^{\mathrm{a}}$ & $845.86 \pm 8.49^{\mathrm{a}}$ & $748.27 \pm 42.49^{\mathrm{b}}$ & $763.10 \pm 33.99^{\mathrm{b}}$ \\
\hline & Bilirubin (mg/dl) & $0.44 \pm 0.01^{\mathrm{a}}$ & $0.00 \pm 0.00^{\mathrm{b}}$ & $0.00 \pm 0.00^{\mathrm{b}}$ & $0.00 \pm 0.00^{\mathrm{b}}$ & $0.00 \pm 0.00^{\mathrm{b}}$ \\
\hline & Hepatic proteins $(\mathrm{mg} / \mathrm{g})$ & $68.31 \pm 3.45^{\mathrm{a}}$ & $69.9 \pm 5.65^{\mathrm{a}}$ & $61.58 \pm 2.44^{\mathrm{a}}$ & $42.28 \pm 3.77^{\mathrm{c}}$ & $28.76 \pm 10.5^{\mathrm{d}}$ \\
\hline & Kidney proteins $(\mathrm{mg} / \mathrm{g})$ & $0.47 \pm 0.04^{\mathrm{a}}$ & $0.40 \pm 0.20^{\mathrm{b}}$ & $0.41 \pm 0.11^{\mathrm{b}}$ & $0.41 \pm 0.02^{\mathrm{b}}$ & $0.40 \pm 0.02^{\mathrm{b}}$ \\
\hline & Heart proteins (mg/g) & $0.19 \pm 0.15^{\mathrm{b}}$ & $0.17 \pm 0.01^{\mathrm{bc}}$ & $0.16 \pm 0.04^{\mathrm{bc}}$ & $0.25 \pm 0.07^{\mathrm{a}}$ & $0.26 \pm 0.18^{\mathrm{a}}$ \\
\hline & Lung proteins $(\mathrm{mg} / \mathrm{g})$ & $2.53 \pm 0.21^{\mathrm{a}}$ & $1.53 \pm 0.22^{\mathrm{b}}$ & $1.54 \pm 0.41^{\mathrm{b}}$ & $1.63 \pm 0.38^{\mathrm{b}}$ & $1.88 \pm 0.46^{\mathrm{b}}$ \\
\hline & Testes proteins $(\mathrm{mg} / \mathrm{g})$ & $0.40 \pm 0.02^{\mathrm{a}}$ & $0.25 \pm 0.14^{\mathrm{b}}$ & $0.20 \pm 0.00^{\mathrm{c}}$ & $0.15 \pm 0.05^{\mathrm{c}}$ & $0.12 \pm 0.14^{\mathrm{d}}$ \\
\hline
\end{tabular}




\begin{tabular}{|c|c|c|c|c|c|c|}
\hline \multirow{20}{*}{ 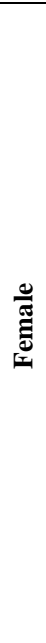 } & Serum creatinine $(\mathrm{mg} / \mathrm{dl})$ & $1367.69 \pm 141.52^{b}$ & $2001.50 \pm 0.00^{\mathrm{a}}$ & $1901.43 \pm 518.93^{\mathrm{a}}$ & $1601.20 \pm 283.05^{\mathrm{a}}$ & $1801.35 \pm 188.70^{\mathrm{a}}$ \\
\hline & Creatinine clearance $(\mathrm{mg} / \mathrm{dl})$ & $0.0017 \pm 0.00016^{\mathrm{a}}$ & $0.0013 \pm 0.00015^{\mathrm{b}}$ & $0.0015 \pm 0.00005^{\mathrm{b}}$ & $0.0005 \pm 0.00002^{c}$ & $0.0006 \pm 0.00000^{\mathrm{c}}$ \\
\hline & Total cholesterol (mg/dl) & $55.35 \pm 4.30^{\mathrm{b}}$ & $55.35 \pm 4.05^{\mathrm{b}}$ & $75.35 \pm 1.42^{\mathrm{a}}$ & $76.82 \pm 1.73^{\mathrm{a}}$ & $51.07 \pm 1.48^{\mathrm{b}}$ \\
\hline & HDL cholesterol (mg/dl) & $31.98 \pm 6.46^{\mathrm{a}}$ & $17.18 \pm 1.49^{\mathrm{b}}$ & $14.89 \pm 0.76^{\mathrm{b}}$ & $15.00 \pm 0.56^{\mathrm{b}}$ & $33.80 \pm 1.12^{\mathrm{a}}$ \\
\hline & LDL cholesterol (mg/dl) & $16.45 \pm 1.25^{\mathrm{c}}$ & $18.01 \pm 0.20^{\mathrm{c}}$ & $45.17 \pm 0.65^{\mathrm{a}}$ & $28.14 \pm 0.58^{\mathrm{b}}$ & $27.64 \pm 1.54^{\mathrm{b}}$ \\
\hline & Triglycerides (mg/dl) & $68.69 \pm 9.17^{\mathrm{a}}$ & $69.33 \pm 2.74^{\mathrm{a}}$ & $61.12 \pm 1.67^{\mathrm{a}}$ & $42.95 \pm 3.31^{\mathrm{b}}$ & $28.76 \pm 2.02^{\mathrm{c}}$ \\
\hline & ALT (U/L) & $45.23 \pm 2.23^{\mathrm{a}}$ & $46.26 \pm 1.23^{\mathrm{a}}$ & $28.80 \pm 6.17^{\mathrm{b}}$ & $32.61 \pm 0.78^{\mathrm{b}}$ & $48.38 \pm 0.68^{\mathrm{a}}$ \\
\hline & AST (U/L) & $52.14 \pm 2.13^{\mathrm{cd}}$ & $48.45 \pm 3.30^{\mathrm{d}}$ & $57.58 \pm 2.11^{\mathrm{c}}$ & $96.03 \pm 0.00^{\mathrm{a}}$ & $73.88 \pm 4.38^{\mathrm{b}}$ \\
\hline & Serum proteins $(\mathrm{mg} / \mathrm{dl})$ & $6.53 \pm 0.87^{\mathrm{b}}$ & $8.66 \pm 0.39^{\mathrm{a}}$ & $6.70 \pm 1.35^{\mathrm{ab}}$ & $6.48 \pm 1.20^{\mathrm{b}}$ & $5.82 \pm 0.21^{\mathrm{b}}$ \\
\hline & Urinary proteins $(\mathrm{mg} / \mathrm{dl})$ & $0.07 \pm 0.00^{\mathrm{b}}$ & $0.06 \pm 0.01^{\mathrm{bc}}$ & $0.08 \pm 0.00^{\mathrm{b}}$ & $0.10 \pm 0.02^{\mathrm{a}}$ & $0.05 \pm 0.00^{\mathrm{c}}$ \\
\hline & Serum glucose $(\mathrm{mg} / \mathrm{dl})$ & $23.40 \pm 6.17^{\mathrm{b}}$ & $19.64 \pm 1.96^{\mathrm{b}}$ & $43.55 \pm 0.69^{\mathrm{a}}$ & $48.80 \pm 0.42^{\mathrm{a}}$ & $46.96 \pm 2.01^{\mathrm{a}}$ \\
\hline & Urinary glucose $(\mathrm{mg} / \mathrm{dl})$ & $10.39 \pm 1.80^{\mathrm{c}}$ & $8.73 \pm 1.37^{\mathrm{c}}$ & $37.14 \pm 10.65^{\mathrm{b}}$ & $30.07 \pm 4.78^{\mathrm{b}}$ & $41.66 \pm 12.60^{\mathrm{a}}$ \\
\hline & Serum urea $(\mathrm{mg} / \mathrm{dl})$ & $50.00 \pm 4.72^{\mathrm{b}}$ & $26.55 \pm 0.94^{\mathrm{c}}$ & $37.93 \pm 0.00^{\mathrm{c}}$ & $31.03 \pm 0.00^{\mathrm{d}}$ & $25.51 \pm 2.83^{\mathrm{a}}$ \\
\hline & Urinary urea (mg/dl) & $336.55 \pm 2.83^{\mathrm{c}}$ & $681.03 \pm 109.87^{\mathrm{b}}$ & $983.79 \pm 76.49^{b}$ & $667.24 \pm 99.15^{\mathrm{b}}$ & $895.51 \pm 79.32^{\mathrm{a}}$ \\
\hline & Bilirubin(mg/dl) & $0.70 \pm 0.070^{\mathrm{a}}$ & $0.00 \pm 0.00^{\mathrm{b}}$ & $0.00 \pm 0.00^{\mathrm{b}}$ & $0.00 \pm 0.00^{\mathrm{b}}$ & $0.00 \pm 0.00^{\mathrm{b}}$ \\
\hline & Hepatic protein $(\mathrm{mg} / \mathrm{g})$ & $68.69 \pm 9.17^{\mathrm{c}}$ & $69.33 \pm 2.74^{\mathrm{c}}$ & $48.50 \pm 0.70^{\mathrm{d}}$ & $89.14 \pm 0.53$ & $76.00 \pm 7.27^{\mathrm{ab}}$ \\
\hline & Kidney proteins (mg/g) & $0.90 \pm 0.04^{\mathrm{a}}$ & $0.37 \pm 0.01^{\mathrm{bc}}$ & $0.43 \pm 0.12^{\mathrm{b}}$ & $0.31 \pm 0.07^{\mathrm{c}}$ & $0.30 \pm 0.01^{\mathrm{c}}$ \\
\hline & Heart proteins (mg/g) & $0.36 \pm 0.02^{\mathrm{a}}$ & $0.22 \pm 0.01^{\mathrm{b}}$ & $0.11 \pm 0.05^{\mathrm{c}}$ & $0.02 \pm 0.00^{\mathrm{d}}$ & $0.09 \pm 0.04^{\mathrm{c}}$ \\
\hline & Lung proteins (mg/g) & $2.49 \pm 0.61^{\mathrm{a}}$ & $0.54 \pm 0.08^{b}$ & $0.66 \pm 0.05^{\mathrm{b}}$ & $0.51 \pm 0.00^{\mathrm{b}}$ & $0.60 \pm 0.15^{\mathrm{b}}$ \\
\hline & Ovary proteins $(\mathrm{mg} / \mathrm{g})$ & $6.09 \pm 0.17^{\mathrm{a}}$ & $3.52 \pm 0.56^{\mathrm{b}}$ & $3.44 \pm 0.00^{\mathrm{b}}$ & $2.04 \pm 0.00^{\mathrm{c}}$ & $0.57 \pm 0.02^{\mathrm{d}}$ \\
\hline
\end{tabular}

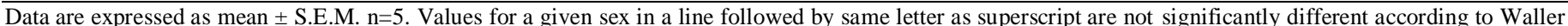

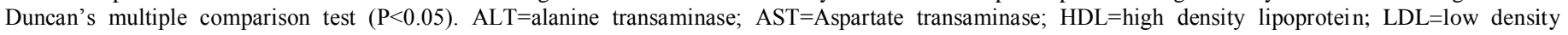
lipoprotein.

\section{DISCUSSION}

The administration of $H$. madagascariensis bark methanol extract by oral route at $5000 \mathrm{mg} / \mathrm{kg}$ did not result in any death; thus, the $\mathrm{LD}_{50}$ is greater than $5000 \mathrm{mg} / \mathrm{kg}$. The extract can be considered as almost non toxic for single administration by oral route (Delongeas et al., 1983). However, signs of toxicity were observed within 3 hours following administration including decrease sensitivity to stimuli (pain and noise) and reduced mobility. The decrease in sensitivity to stimuli could result from nerve damage by desensitizing nerve receptor cells (Bep, 1986) while decrease mobility may reflect the sedative or tranquilizing effect of the extract (Gatsing et al., 2005).

In sub-acute treatment, $H$. madagascariensis extract resulted in animals of both sexes an increase in white blood cell counts at $200 \mathrm{mg} / \mathrm{Kg}$ particularly eosinophils and lymphocytes. An increase in these parameters may indicate the activation of the immune system, inflammation, necrosis or a malignant infection (Marieb and Hoehn, 1999). Low neutrophils and monocytes levels were observed from $200 \mathrm{mg} / \mathrm{kg}$ in animals of both sexes. Similar results were observed with lymphocytes and eosinophils beyond $200 \mathrm{mg} / \mathrm{kg}$ on females. The decrease of these parameters conveys the adverse effect of the extract on the latter. Eosinophils and neutrophils are involved in innate immunity mechanisms. Eosinophils are activated in response to parasites aggression and allergic reactions. Neutrophils are involved in the process of phagocytosis and inflammatory reaction (Lewis et al., 2011). The H. madagascariensis extract could therefore compromised innate immune response by either inhibiting any of the steps of the differentiation of these cells or act directly on the cells.

A decrease in red blood cell levels in males and females was observed. Red blood cells are involved in the transport of respiratory gases within the body and in the maintenance of acidbase balance. A decrease in their rate could result in hypoxia or myocardial infarction (Marieb and Hoehn, 1999). However, hemoglobin concentration, the more sensitive parameter to detect anemia was not affected. Therefore the administration of this extract could not present a risk of anemia. Additionally, $H$. madagascariensis extract caused a decrease in blood platelet levels in both sexes. Platelets are small irregularly shaped fragments which freely circulate in blood. They are involved in the clotting process and their reduction could result in increased bleeding risk (Alberts et al., 2007).Thus, the methanol extract of $H$. madagascariensis bark could therefore have anticoagulant properties.

An increase in the total cholesterol concentration, LDL cholesterol and a decrease in triglycerides and HDL cholesterol concentrations from $200 \mathrm{mg} / \mathrm{kg}$ were observed in animals of both sexes. These indicate a disturbance of lipid metabolism following the administration of methanol extract of $H$. madagascariensis in sub-acute treatment. Additionally, H. madagascariensis extract could therefore have hyperlipidemic property at high-dose. This could lead to heart disease risk, as it is well established that LDL cholesterol promotes the development of fatty deposits and atheroma on the arteries leading to cardiovascular disease. Previous studies on toxicity study of $H$. madagascariensis aqueous leaves extract by oral route revealed hyperlipidemic properties up to $200 \mathrm{mg} / \mathrm{kg}$ (Kengni et al., 2016).

There was a decrease in glycaemia at $200 \mathrm{mg} / \mathrm{kg}$ and increase glycaemia and glycosuria from $400 \mathrm{mg} / \mathrm{kg}$ in animals of both sexes was observed, indicating the risk of developing diabetes after prolonged use at high doses of the $H$. madagascariensis methanol extract. These results are in line with Maffo, (2007) who reported hypoglycemic properties of $H$. madagascariensis leaves at $200 \mathrm{mg} / \mathrm{kg}$.

A decrease in transaminase activity from $400 \mathrm{mg} / \mathrm{kg}$ was recorded. Transaminases are tissue enzymes, catalyzing the transport of alpha-amino groups of alanine and aspartic acid at the 
alpha-ketoglutaric acid. They are synthesized in the cytoplasm and released into circulation when cells are damaged (Ozturk et al., 2009). The activity of these enzymes is elevated in case of myopathy or myocardial infarction. ALT is more specific to liver damage, while the AST are slightly more sensitive (Peirs, 2005). The decreased activity of these enzymes could express the hepatoprotective property (Peirs, 2005). These results are in line with Adeneye et al. (2008) who reported that pretreatments with $100-500 \mathrm{mg} / \mathrm{kg}$ of $H$. madagascariensis aqueous roots extract significantly attenuated the elevations of ALT, AST and other biochemical parameters in acute and repeated dose acetaminophen induced hepatotoxicity in rats. But, Kengni et al. (2016) and Biapa et al. (2012) results stated hepatoxicity of aqueous leaves and hydroalcoholic bark extract of $H$. madagascarienis respectively. This poses the choice of medicinal plant part and extraction solvent for a medical operation. Moreover, a need for standardization of plant extracts since phytochemical composition varies according to external factors including climate, soil and time.

Bilirubin is a yellow pigment which results from degradation of hemoglobin and other heme proteins (cytochromes, catalase ...). Its abnormal accumulation in blood and tissues lead to jaundice. The cancellation of the concentration of total bilirubin from $200 \mathrm{mg} / \mathrm{Kg}$ in the treated animals might suggest the activity of the extract against liver cirrhosis and severe hepatitis. The results obtained fit with hepatoprotective effect observed and histological analysis sections of the liver which revealed vascular congestion that could be assign to a vasoconstrictor action of the extract on blood vessel walls or stress of the animals during sacrifice. However, these results are contrary to those reported by Kengni et al. (2016) who found increase in serum transaminases activity when administering aqueous extract leaves of $H$. madagascariens in sub-acute treatment by oral route.

Increase in proteinuria and glycosuria from $400 \mathrm{mg} / \mathrm{kg}$ in animals of both sexes was noted. In Parallel to these effects, extract spawned an increase in the rate of elimination of creatinin from $200 \mathrm{mg} / \mathrm{Kg}$ not associated with increase serum creatinin. On the other hand, the decrease in serum urea level associated with an increased urinary elimination was observed. Moreover, the analysis of histological sections of the kidneys revealed a narrowing of the urinary tract. These activities reflect each, an improvement in renal function following administration in subacute treatment with the extract of $H$. madagascariensis. Consequently, the methanol extract of $H$. madagascariensis stem bark may inhibit tubular reabsorption of these molecules.

The increase in serum protein levels associated with decreasing levels of some organelle proteins including kidneys, lungs, ovaries and testes could be a glint of the toxic effect of methanol extract of $H$. madagascariensis on these organs. The results are in line with histology which revealed necrosis.

Toxic effects observed in hematological parameters (neutrophils and eosinophils), biochemical parameters (HDL cholesterol) and histology of liver and kidney in both sexes were remedied 15 days after treatment, suggesting possible reversibility of the toxic effects of the extract.

\section{CONCLUSION}

The methanol extract from the stem bark of $H$. madagascariensis administered orally has a $\mathrm{LD}_{50}$ higher than 5000 $\mathrm{mg} / \mathrm{kg}$ in acute toxicity and hence is practically not toxic. In subacute treatment, increase in the number of lymphocytes and eosinophils was observed while a decrease in the number of polynuclear neutrophils, monocytes, red blood cells and platelets was noted. Biochemical parameters analysis showed an increase in total cholesterol, LDL cholesterol, glycaemia and glycosuria, and a decrease in triglycerides, HDL cholesterol, bilirubin and transaminases activity. However, an increase proteinuria, glucosuria and speed of elimination of creatinin were also observed. Histological study revealed a beginning of vascular congestion in the liver, a narrowing of the urinary tract and expansion of mesangium in kidney, necrosis of cardiac muscle fibers and sexual organs. This study provides valuable data on the acute and sub-acute oral toxicity profiles of $H$. madagascariensis stem bark that should be very useful for any future in vivo and clinical studies of the stem bark of this plant in medicine.

\section{Abbreviations}

LDL: light density lipoproteins, HDL: high density lipoproteins, MIC: minimum inhibitory concentrations, ALT: Alanine transaminase, AST: Aspartate transaminase, EDTA: Ethylenediaminetetraacetic acid, DMSO: Dimethyl sulfoxide.

\section{Acknowledgements \\ Authors acknowledge jean Pierre Zoobo for english revision.}

Conflict of Interests: There are no conflicts of interest.

\section{REFERENCES}

Adeneye AA, Olagunju JA, Elias SO, Olatunbosun DO, Mustafa AO, Adeshile OI, Ashaolu AO, Laoye TA, Bamigboye AO, Adeoye AO. Harungana madagascariensis in acute and repeated acetaminophen hepatotoxic rats. Int J Appl Res Na Prod, 2008; 1(3): 2942 .

Alberts B, Johnson A, Lewis J, Raff M, Roberts K, Walter P. Leukocyte functions and percentage breakdown, Molecular biology of the cell. $5^{\text {th }}$ ed. New-York: Garland Science; 2007.

Anguinaga YJ, Claudiano SG, Marcusso FP, Ikefuti C, Ortega GG, Eto FS, Cruz da C. Acute toxicity and determeination of the active constituents of aqueous extract of Uncaria tomentosa barh in hyphessobrycon eques. Journal of toxicology, 2014.

Bep O-B. Medical plants in tropical West Africa. $1^{\text {st }}$ ed, Cambridge University Press; 1986.

Biapa NPC, Oben EJ, Ngogang YJ. Acute and sub-acute toxicity of Harungana madagascariensis LAM. AJPSP, 2012; 3(1): 45-57.

Bouiddouh Z. Evolution des paramètres biochimiques sériques chez les rats Wistar traités par l'extrait éthanolique des graines de la coloquinte (Citrullus colocynthis). Master thesis, Université d'Abou Bakr Belkaid -Tlemcen, Algérie ; 2012. 
Delongeas JL, Burnel D, Netter P, Grignon M, Mur JM. Royer RJ, Grignon G. Toxicité et pharmacocinétique de l'oxychlorure de zirconium chez la souris et le rat. J Pharmacol, 1983; 14(4): 437-47.

Etchike CA, Sassa AM, Abakar A, Nyonbourg E. Evaluation in vitro de l'activité antibactérienne de cinq plantes de la pharmacopée traditionnelle de l'Adamaoua (Cameroun). CAJEB, 2011 ; 7(1): 22-7.

Gatsing D, Aliyu R, Kuiate JR, Garba IH, Jaryum KH, Tedongmo N, Tchouanguep FM, Adoga GI. Toxicological evaluation of the extract of Allium sativum bulbs on laboratory mice and rats. CAJEB, 2005; 1(1): 39-45.

Kengni F, Fodouop SPC, Tala SD, Djimeli NM, Fokunang C, Gatsing D. Antityphoid properties and toxicity evaluation of Harungana madagascariensis Lam (Hypericaceae) aqueous leaf extract. J Ethnopharmacol, 2016 ; 6(2): 62-76.

Koné MW, Kamanzi AK. Inventaire ethnomédical et évaluation de l'activité anthelminthique des plantes médicinales utilisées en Côte d'Ivoire contre les helminthiases intestinales. Pharm Méd Trad Afr, 2006; $14: 55-72$.

Lewis SL, Dirksen SR, Heitkemper MM, Bucher L, Camera IM. Soins infirmiers - médecine chirurgie. $8^{\mathrm{e}}$ ed, Tome 1, Canada ; 2011.

Maffo S. Evaluation des propriétés antihyperglycémiante et hypolipidémiante in vivo des fractions polysaccharidiques solubles de deux plantes médicinales camerounaises Chromolaena odorata et Harungana madagascariensis. Master thesis, University of Yaounde I; 2007.

Mangambu M. Contribution à l'étude phytochimique de quelques plantes médicinales antidiabétiques de la ville de Bukavu et ses environs (Sud-Kivu, R. D. Congo). J Appl Biosci, 2014 ; 75: 6211-20.

Manzo M. Etude phytochimique et pharmacologique de plantes antipaludiques utilisées en médecine traditionnelle congolaise. Thèse de Docteur en Sciences Biomédicales et Pharmaceutiques, Université de Liège, Université de Kinshasa; 2012.

Marieb EN, Hoehn KN. Human Anatomy and Physiology. $9^{\text {th }}$ ed, USA; 1999

Mouokeu RS, Ngono NRA, Lunga PK, Koanga MM, Tiabou TA, Njateng GSS, Tamokou JDD, Kuiate JR. Antibacterial and dermal toxicological profiles of ethyl acetate extract from Crassophalum bauchiense (Hutch.) Milne-Redh (Asteraceae). BMC Complementary and Alternative Medicine, 2011; 11:43.

Naidu Rj, Ismail R, Sasidharan S. Acute oral toxicity and brine shrimp lethality of methanol extract of Mentha spicata 1 (lamiaceae). Trop J Pharm Res, 2014;13(1):101.

Nicolas J. Plantes médicinales du nord de Madagascar ethnobotanique antakarana et informations scientifiques. Jardins du Monde, 2012 ; 295: 134-5.

$\mathrm{OCDE}_{\mathrm{a}}$. Lignes directrices de l'OCDE pour les essais de produits chimiques, Toxicité orale aigue-Méthode de l'ajustement des doses ; 2008

$\mathrm{OCDE}_{\mathrm{b}}$. Lignes directrices de l'OCDE pour les essais de produits chimiques, études de toxicité orale à dose répétée pendant 28 jours sur les rongeurs ; 2008.

Ozturk N, Lee JH, Gaddameedhi S, Sancar A. Loos of crytochrome reduces cancer risk in p 53 mutant mice. Proc Natl Acad Sci, 2009; 106(8): 2841-46.

Peirs C. Contribution à l'étude phytochimique de Galega officinalis $L$ (Fabaceae). Pharmacy thesis, Laboratoire Pharmacochimie des Substances Naturelles et Pharmacophores Redox, UMR 152, Faculté des Sciences Pharmaceutiques, Toulouse : France ; 2005.

\section{How to cite this article:}

Etame RME, Mouokeu RS, Ngane RAN, Assam JP, Masoohe AM, Tchientcheu R, Hopogap MLM, Etoa FX. Acute and sub-acute toxicity of Harungana madagascariensis LAM (Hypericaceae) stem bark methanol extract. J App Pharm Sci, 2017; 7 (03): 160167. 